\title{
COVID-19 Vaccine Uptake and Associated Factors Among Health Professionals in Ethiopia
}

\section{Dufera Rikitu Terefa \\ Adisu Tafari Shama \\ Bikila Regassa Feyisa (D) \\ Adisu Ewunetu Desisa \\ Edosa Tesfaye Geta \\ Melese Chego Cheme \\ Afework Tamiru Edosa}

Department of Public Health, Institute of Health Science, Wollega University,

Nekemte, Ethiopia
Correspondence: Dufera Rikitu Terefa Department of Public Health, Institute of Health Science, Wollega University, P.O.Box $=395$, Nekemte, Ethiopia Tel +25I-922260706

Email duferarikitu24@gmail.com; duferar@wollegauniversity.edu.et
Background: Ethiopia has received 2.2 million doses of COVID-19 vaccine from the COVID-19 Vaccines Global Access (COVAX) facility and planned to vaccinate $20 \%$ of its population by the end of 2021. However, evidence on the current uptake of the vaccine in our country is scanty. Therefore, this study aimed to assess COVID-19 vaccine uptake and associated factors among health professionals in Ethiopia.

Methods: A national online cross-sectional E-survey was conducted on COVID-19 vaccine Uptake and associated factors among health professionals in Ethiopia from June 1 to 30, 2021. A semi-structured questionnaire was created on Google forms and disseminated online. The snowball sampling technique through the authors' network with Ethiopian residents on the popular social media like Facebook, telegram, and email was used. Descriptive statistics were performed. Multivariable logistic regression analysis was performed using Statistical Package for Social Sciences version 25, and all variables with P-value $<0.05$ and adjusted odds ratio at $95 \%$ CI were used to declare the predictors of the outcome variable.

Results: A total of 522 health professionals participated in the survey, of which about 324 $(62.1 \%)$ of them were vaccinated with any of the COVID-19 vaccines at least once. The study indicated that COVID-19 vaccine uptake was associated with age range from 35 to 44 years $[\mathrm{AOR}=12.97,95 \% \mathrm{CI}: 2.36-71.21]$, age beyond 45 years $[\mathrm{AOR}=18.95,95 \% \mathrm{CI}=$ 2.04-36.29], being male [AOR $=2.91,95 \% \mathrm{CI}=1.05,8.09]$, being only an academician [AOR $=0.23,95 \% \mathrm{CI}: 0.10-0.49]$, academicians working in University hospitals [AOR = $0.19,95 \%$ CI: $0.05-0.83$ ], perceiving their family as healthy [AOR $=4.40,95 \% \mathrm{CI}: 2.21-$ 8.75], no history of receiving other vaccine before as an adult $[\mathrm{AOR}=4.07,95 \% \mathrm{CI}: 2.07$ $8.01]$ and no history of contact with confirmed COVID-19 patients or clients [AOR $=0.42$, 95\% CI: $0.20-0.86]$.

Conclusion: The study found that COVID-19 vaccine uptake among health professionals was low. This was not sufficient to achieve herd immunity as at least nine out of ten health professionals are required for herd immunity. Ages, sex, place of work, perceived family health status, previous experience of receiving a vaccine as an adult and history of contact with COVID-19 clients or patients were the factors that influence the vaccine uptake among health professionals in Ethiopia. Hence, decision makers and health managers should consider instituting mandatory vaccination for health professionals and design strategies for the provision of the vaccine.

Keywords: COVID-19, vaccine, health professionals, uptake, Ethiopia

\section{Plain Language Summary}

COVID-19 Vaccine uptake is the number of people vaccinated with a certain dose of the vaccine in a certain time period, which can be expressed as an absolute number or as the 
proportion of a target population. Although Ethiopia has planned to vaccinate $20 \%$ of its population with the COVID-19 vaccine by the end of 2021, evidence on the current uptake among health professionals was scanty. Therefore, this study aimed to assess COVID-19 vaccine uptake and associated factors among health professionals in Ethiopia. This finding revealed that vaccine uptake was not sufficient as health professionals were the vital source of information and their role in promoting COVID-19 vaccine uptake is crucial. Hence, decision makers and health managers should consider instituting mandatory vaccination for health professionals and design strategies for the provision of the vaccine.

\section{Background}

Coronavirus disease 2019 (COVID-19) is caused by Severe Acute Respiratory Syndrome Corona virus-2 (SARS-CoV-2). ${ }^{1}$ First identified in Wuhan, China at the end of December 2019, ${ }^{2}$ COVID-19 was declared as a Public Health Emergency of International Concern by the World Health Organization (WHO). ${ }^{3}$ Globally, it affected 235,413,296 individuals and caused 4,809,246 deaths until October 4, 2021. ${ }^{4}$ Ethiopia confirmed its index case on March 13, 2020, ${ }^{5}$ and as of October 3, 2021, there were 348,669 cases and 5722 deaths in the country. ${ }^{6}$ In addition to the effective public health measures such as educating the general population, social distancing, wearing face masks, hand washing, and avoidance of crowded conditions, COVID-19 vaccination has emerged and currently 22 vaccines were approved for use. $^{7,8}$ Accordingly, about $47 \%$ and $35 \%$ of the world populations were vaccinated for COVID-19 partly and fully, respectively, until October 4, 2021. ${ }^{9}$ Ethiopia received the initial 2.2 million doses of vaccine from the COVID-19 Vaccines Global Access (COVAX) facility and planned to vaccinate $20 \%$ of its population with COVID19 vaccine by the end of $2021 .{ }^{10}$

Vaccine uptake is the number of people vaccinated with a certain dose of the vaccine in a certain time period, which can be expressed as an absolute number or as the proportion of a target population. ${ }^{11}$ The WHO and the Centers for Disease Control and Prevention (CDC) have identified health-care workers (HCWs) as a population with significantly increased risk of infection from the SARS-CoV-2, and Phase 1 allocation of COVID-19 vaccine targeted HCWs to protect themselves and the public health. ${ }^{12-14}$ Moreover, health professionals serve as a trusted source of information on public health topics and their role in promoting COVID-19 vaccine uptake is crucial. Hence, regarding a novel COVID-19 vaccine uptake, they need to avoid refusal to inspire attitude of the general public towards a positive one. ${ }^{15}$

Health professionals showed skepticism towards vaccine uptake, ${ }^{16}$ and $22.51 \%$ of health-care workers hesitate over COVID-19 vaccination worldwide. ${ }^{17}$ In the Democratic Republic of Congo, only $27.7 \%$ of HCWs said that they would get vaccinated if the COVID-19 vaccine was available. ${ }^{18}$ A large proportion $(47.32 \%)$ of the Ethiopian population are unwilling to take the COVID19 vaccine while $21.31 \%$ are not decided. ${ }^{19}$ Despite the similarity in the estimate of the rates of COVID-19 vaccination hesitancy in health professionals and the general population, ${ }^{20}$ sometimes health-care workers become more hesitant to COVID-19 vaccine than the general population $(62.5 \%$ vs. $22.2 \%){ }^{21}$

By nature of their occupation, health-care workers are at an increased risk of exposure to the severe acute respiratory syndrome corona virus 2 (SARS-COV2), and vaccine hesitancy can affect their lives more. ${ }^{22}$ Though, globally many countries are under reporting death of health-care workers due to COVID-19, health-care workers are dying every 30 minutes and more than 17,000 health-care workers died in the last one year as Amnesty report of March 5, $2021 .^{23}$ In addition to the possibility of contaminating their families and patients, hesitancy of health professionals could lead to hesitancy of the general public at large as health professionals are trusted sources of information on the issues of public health, including COVID-19 vaccines. ${ }^{15}$ If health professionals continue to remain hesitant towards COVID-19 vaccines, they cannot be in a position to recommend these vaccines to the general public and ensure mass vaccinations with the available COVID-19 vaccines. ${ }^{17}$

So far, the studies conducted identified that low vaccine uptake was influenced by perceptions and misconceptions about vaccine efficacy, adverse effects, and vaccinations causing disease. ${ }^{16,24-27}$ Effective use of vaccination was seen, ${ }^{16,24,27}$ desire to protect themselves and their family and friends, ${ }^{25}$ and vaccination recommendation from a family member or close friends. ${ }^{28}$ Among the general public for all vaccines, receiving a vaccination recommendation by a trusted medical provider has a significant influence on vaccination acceptance. ${ }^{28,29}$ A study conducted in Ethiopia found that only a small percent of the population was willing to accept the COVID-19 vaccine, and most people were hesitating about the vaccine. Also, almost all reported that health workers 
should be vaccinated first. ${ }^{19}$ This indicated that good vaccination acceptance among health professionals would enhance the vaccination rate in the general population. However, there was no prior study conducted among health professionals who were expected to be models for the general population toward the vaccine uptake.

As far as the knowledge of the authors is concerned, no prior study was conducted on COVID-19 vaccine uptake and associated factors among health professionals in the study area. Therefore, this study aimed to assess COVID19 vaccine uptake and associated factors among health professionals in Ethiopia.

\section{Methods and Materials}

\section{Study Area and Period}

The study was conducted in the Federal Democratic Republic of Ethiopia from June 1 to 30, 2021. Ethiopia is the largest and the most populated country that is located in the horn of Africa.

Currently, the Ethiopian health system has three tiers of service having health facilities of different types. As of today, the country has a total of over 73,514 health professionals working in 24,000 health facilities of all types; 392 government hospitals, 4064 health centers, 16,989 health posts. The numbers of operational private facilities are 5401 primary clinics, 62 hospitals, 536 specialty clinics and 1308 medium clinics providing promotive, preventive, and curative health services to the population. Over 3000 medical professionals were operating in different disciplines in 2019 in Addis Ababa.

\section{Study Design}

A national online cross-sectional E-survey was conducted on COVID-19 Vaccine Uptake and associated factors among Health Professionals in Ethiopia, 2021.

\section{Source Population}

All employed health professionals who work in health institutions of Ethiopia were the source population.

\section{Study Population}

All those selected or surveyed employed health professionals working in the health institutions of Ethiopia were the study population.

\section{Eligibility Criteria}

Employed health professionals aged 18 or more years, who reside in Ethiopia, who were able to read and understand the English language, agreed to participate and completed the survey were included in the analysis. Those health professionals who could not access the Internet were excluded. Also, incomplete responses were excluded from the analysis.

\section{Sample Size Determination}

A total of 522 respondents participated in the study across the country within the study period as it is difficult to predetermine the sample size for the survey.

\section{Sampling Technique and Procedures}

During this study, Ethiopia was in a partial lockdown with different institutions due to fear of COVID-19. Hence, we opted to use popular social media for enrolling potential participants. For this, we employed snowball sampling where we identified all the existing health professionals in the country. Google Form link to the questionnaire was then sent to the potential participants via the identified social medias.

\section{Study Variables}

COVID-19 vaccine uptake was the outcome variables measured in the study, whereas various factors described in four main sections such as; socio-demographic and economic variables (age, sex, religion, ethnicity, residence, marital status, educational status, family size and monthly income); profession and work-area-related variables (place of work or types of facility, types of profession); health status and exposure variables (perceived own health status, perceived family health status, tested for covid-19, history of chronic illness, history of vaccination for other diseases and contact history with COVID-19 patients or clients) were the independent variables.

COVID-19 vaccine uptake among health professionals was measured using the question: "Have you taken COVID-19 vaccine at least one dose as of today?". This question was answered as either "Yes or No" type closeended question to indicate the vaccine uptake.

\section{Operational Definitions}

Vaccine Uptake: Is an absolute number of people who have received a specified vaccine dose (ie, at least one dose of a COVID-19 vaccine) during the period of the survey. It was measured by the closed-ended question as "Have you been vaccinated with any of COVID-19 vaccines at least once currently?" (Yes/No). Those participants who have taken the vaccine answered as "Yes" and 
those who were not vaccinated answered as "No" to the question to indicate the vaccine uptake. ${ }^{30}$

Health Institutions: Are public and private organizations that provide health care and related services to the provision of patients and clients at different levels in the health system of Ethiopia (health facilities, health offices from district to $\mathrm{FMOH}$ and academic institutions).

Health Facilities: Are public facilities at different levels of health care, according to the Ethiopian current health tier system (three tier systems), where health services are provided (primary level, secondary level and tertiary level health care) and private health facilities of all types. $^{31}$

Health Professionals: A physician or other health-care practitioners licensed, certified or registered to perform specific health-care services in public and private health institutions consistent with the state law.

Perceived Health Status: The respondent's report about their or family health status, which was assigned with numerical values according to the scale: very $\operatorname{good}=5$, $\operatorname{good}=4$, medium $=3$, poor $=2$ and very poor $=1$; then the value was re-categorized into Healthy and Not healthy.

Chronic Illnesses: is a disease condition that lasts more than 3 months on respondents or their family members.

\section{Data Collection Instrument}

Data was collected via an online platform using a semistructured questionnaire. The questionnaire was adapted by reviewing different literates and modified to the local context. $^{32}$ The questionnaire was prepared in English and comprised of three main sections including sociodemographic and socio-economic factors; profession and work-area-related factors and health status and exposurerelated factors.

The questionnaire contains a brief introduction to the study's background, objective of the study, eligibility criteria, voluntary nature of participation, declaration of confidentiality, and anonymity and informed consent of each participant asking whether or not they want to participate in this study. Participants must respond positively for further proceeding with the self-reporting on the questionnaire link on the Google form.

\section{Data Collection Procedures}

During data collection, study participants were recruited using snowball sampling technique from health professionals through the author's network with residents on the popular social media such as Facebook, Telegram, and email. The link of the questionnaire was posted by the authors on the above-mentioned Social Media and to fulfill the terms and conditions on the link. Regarding the responses of respondents' terms and conditions of Google forms were first assured before proceeding to the survey.

\section{Data Quality Control}

Different measures were undertaken to maintain the quality of the data before, during and after data collection. Before the actual data collection, the questionnaire prepared in English was translated into the local language and then translated back into English, and the contents of the questionnaires were checked for its consistency. Also, the questionnaire was pretested on some of the health professionals surrounding us, and then after feedbacks have been received from those participants, some modifications and corrections were made accordingly to the questionnaire before distributing to the whole. Finally, incomplete responses were excluded from the analysis.

\section{Data Processing and Analysis}

Completed questionnaires were extracted from Google Forms and exported to a Microsoft Excel 2013 for coding and then exported to Statistical Package for Social Science (SPSS) version 25 software for cleaning and analysis. Data analysis was performed using a variety of descriptive statistics such as frequency distribution, percentage, and computing mean scores. Then, data was presented using tables, graphs and charts. Association between dependent and independent variables was analyzed first using binary logistic regression analysis. Variables that had $\mathrm{p} \leq 0.25$ on binary logistic regression analysis were considered to be candidates for multivariable logistic regression analysis. Multivariable logistic regression was employed to analyze the relationship between the outcome variable and predictor variables. In multivariable logistic regression, the backward (LR) variable selection method was used in the analysis. Independent variables with P-values $<0.05$ and Adjusted Odds Ratio at $95 \%$ CI were used to declare the predictors of the outcome variable.

Collinearity was checked using a collinearity matrix, with all values of variance inflation factors (VIF) less than 3.5 (cutoff point for VIF $<10$ ). The goodness of fit of the model was assessed using the Hosmer and Lemeshow test, which indicated the non-significant chi-square ( $p$-value of 0.257). This indicates that the model was appropriate for data analysis. 


\section{Ethical Approval and Considerations}

The study was conducted after appropriate research ethical approval was obtained from the ethical review Board of Wollega University, Institute of health sciences (Reference number: IRB/298/2021). This study was conducted in accordance with the Declaration of Helsinki. The questionnaire was designed to be anonymous, and the result did not identify the personality of the respondents, rather it was presented in the aggregated statistics. Data was kept in protected and safe locations. Paper-based data was kept in a locked cabinet and computer-based data was passwordsecured. Data sharing was enacted based on the consent and permission of research participants and the ethical and legal rules of data sharing, and it was not accessed by a third person, except the research teams.

\section{Results}

\section{Characteristics of the Study Participants}

A total of 522 health professionals were involved and completed the survey questionnaire. Most of the respondents were within the age range of 30-39 years, 406 $(77.8 \%)$; with the mean age of $(30.9+\mathrm{SD}=4.75)$. Male constituted $471(90.2 \%)$ of the study participants. Majority of participants belonged to Oromo, $321(61.5 \%)$ by ethnicity; protestant, 168 (32.2\%) by religion and were married, $351(67.2 \%)$. Most of the respondents were Bachelors of Science degree, 225 (43.1\%); followed by Master of Science degree, 197 (37.7\%) holders. The majority of the respondents were urban residents, $448(85.8 \%)$ and 450 $(86.2 \%)$ of them had less than five people per household (Table 1).

Regarding profession and work-area-related characteristics; the highest numbers of participants were from only clinical staffs, $183(35.1 \%)$ followed by only academic staff $160(30.7 \%)$, while academicians working in university hospitals constitute only $23(4.4 \%)$. Of those only academicians, about $67(20.7 \%)$ of them had taken the vaccine.

The place of work for most of the participants was universities or colleges (academicians), 152 (29.1\%). The highest proportion of vaccinated individuals were observed in those who were working in health centers, $90(27.8 \%)$ followed by those who worked in hospitals, $75(23.1 \%)$ and health offices, 82 (25.3\%). Profession wise, most of the participants were public health, 258 (49.4\%) and nurses, 124 (23.8\%). Among those who had participated, $162(50.0 \%), 67(20.7 \%)$ and $27(8.3 \%)$ of involved public health, nurses and medical doctors had taken the vaccine at least one dose, respectively.

Concerning health status and exposure-related characteristics; those respondents who perceived themselves as healthy were about $425(81.4 \%)$, while those who perceive their family as healthy were accounted for as $383(73.4 \%)$. Of these who perceived their family as healthy, 255 (78.7\%) had taken the vaccine.

About eight out of ten, 407 (78\%) respondents had history of receiving other vaccines as an adult for themselves. Of these, $270(83.3 \%)$ had taken the vaccine.

Almost half, 248 (47.1\%) of the respondents had history of contact with confirmed COVID-19 patients or clients. Of these, 125 (23.9\%) and 123 (23.6\%) of them had direct contact and had not direct contact with them, respectively. Whereas, 274 (52.5\%) of the respondents had no contact with confirmed COVID-19 patients and of these almost half, $154(47.5 \%)$ of them had taken the vaccine. Only $99(19 \%)$ of the respondents were involved in the COVD-19 isolation center or care, and of these only 72 $(22.2 \%)$ had taken the vaccine.

\section{COVID-19 Vaccine Uptakes}

Of all health professionals who were involved in the survey, about $324(62.1 \%)$ of them were vaccinated with any of the COVID-19 vaccines at least one dose, whereas about 198 (37.9\%) were not vaccinated or had not taken the vaccine (Table 2).

\section{Factors Associated with COVID-19 Vaccine Uptake}

In the binary logistic regression analysis, some of the variables such as age, sex, residence, educational status, monthly income, place of work, type of profession, staffing or expertise, perceived own health status, perceived family health status, ever been tested for COVID-19, presence of chronic disease for respondent, history of receiving other vaccine before as an adult, ever involved in COVID-19 isolation center or care, history of contact with confirmed COVID-19 patients or clients were statistically associated with COVID-19 vaccine uptake (Table 2). Whereas, some of the variables such as religion, ethnicity, marital status and family sizes were excluded because of their p-value was $>0.25$ on binary logistic regression analysis.

After adjusting for other variables such as age, sex, staffing or expertise, perceived family health status, history of receiving other vaccines before as an adult, history 
Table I Socio-Demographic and Economic Characteristics of the Study Participants Toward COVID-19 Vaccine Uptake and Associated Factors Among Health Professionals in Ethiopia, $202 \mathrm{I}(\mathrm{N}=522)$

\begin{tabular}{|c|c|c|}
\hline Variables & Categories & Frequency (\%) \\
\hline \multirow[t]{4}{*}{ Age } & $18-29$ & $20(3.8 \%)$ \\
\hline & $30-39$ & 406 (77.8\%) \\
\hline & $40-49$ & 71 (13.6\%) \\
\hline & $50+$ & $25(4.8 \%)$ \\
\hline \multirow[t]{2}{*}{ Sex } & Male & 47। (90.2\%) \\
\hline & Female & 51 (9.8\%) \\
\hline \multirow[t]{6}{*}{ Religion } & Orthodox & 135 (25.9\%) \\
\hline & Protestant & $168(32.2 \%)$ \\
\hline & Muslim & $160(30.7 \%)$ \\
\hline & Catholic & $21(4.0 \%)$ \\
\hline & Wakefata & 17 (3.3\%) \\
\hline & Others ${ }^{a}$ & 21 (4.0\%) \\
\hline \multirow[t]{6}{*}{ Ethnicity } & Oromo & 321 (61.5\%) \\
\hline & Amhara & 94 (I8.0\%) \\
\hline & Tigre & $21(4.0 \%)$ \\
\hline & Gurage & 31 (5.9\%) \\
\hline & Somali & $21(4.0 \%)$ \\
\hline & Other ${ }^{b}$ & $34(6.5 \%)$ \\
\hline \multirow[t]{2}{*}{ Resident } & Urban & 448 (85.8\%) \\
\hline & Rural & 74 (14.2\%) \\
\hline \multirow[t]{2}{*}{ Marital status } & $\begin{array}{c}\text { Never married/divorced/ } \\
\text { widowed }\end{array}$ & I7I (32.8\%) \\
\hline & Married & $35 \mathrm{I}(67.2 \%)$ \\
\hline \multirow{4}{*}{$\begin{array}{l}\text { Educational } \\
\text { status }\end{array}$} & College Diploma & $28(5.4)$ \\
\hline & BA/BSc Degree & $225(43.1)$ \\
\hline & MA/MSc Degree & $197(37.7)$ \\
\hline & PHD and above & $72(13.8)$ \\
\hline \multirow[t]{2}{*}{ Family size } & $<5$ & 450 (86.2\%) \\
\hline & $\geq 5$ & 72 (I3.8\%) \\
\hline \multirow[t]{5}{*}{ Monthly income } & $165 \mid-3200$ & $13(2.5 \%)$ \\
\hline & $320 I-5250$ & 44 (8.4\%) \\
\hline & $5251-7800$ & $172(33.0 \%)$ \\
\hline & $780 \mathrm{I}-10,900$ & I7I (32.8\%) \\
\hline & $>10,900$ & $122(23.4 \%)$ \\
\hline
\end{tabular}

Note: ${ }^{a} \mathrm{Hindu}$, Apostolic Christian. ${ }^{\mathrm{b}}$ Burji, Sidama, India (expatriate). of contact with confirmed COVID-19 patients or clients showed statistically significant association with the COVID-19 vaccine uptake in multiple logistic regression analysis (Table 2).

Old age health professionals, 40-49 years were thirteen times more likely to uptake COVID-19 vaccine $[\mathrm{AOR}=12.97$, 95\% CI: $2.36-71.21]$ while those age $>50$ years old were about nineteen times more likely to uptake COVID-19 vaccine $[\mathrm{AOR}=18.95,95 \% \mathrm{CI}=2.04-36.29]$ when compared to younger age, 18-29 years old, health professionals. Male health professionals were 2.91 times more likely to uptake COVID-19 vaccine $[\mathrm{AOR}=2.91,95 \% \mathrm{CI}: 1.05-8.09]$ than their counterparts.

The probability of COVID-19 vaccine uptake for those who were only academic staffs and academic staffs working in university hospitals were reduced by $77 \%$ [AOR = $0.23,95 \%$ CI: $0.10-0.49$ ] and $81 \%$ [AOR $=0.19,95 \% \mathrm{CI}$ : 0.05-0.83] compared to those who were health office staffs.

The odds of COVID-19 vaccine uptake was 4.4 times higher in those who perceived their family health status as healthy $[\mathrm{AOR}=4.40,95 \% \mathrm{CI}: 2.21-8.75]$ compared to their counterparts. The study revealed that health professionals who did not have a history of receiving other vaccines before as an adult were about 4 times more likely to uptake COVID-19 vaccine [AOR $=4.07,95 \%$ CI: $2.07-$ $8.01]$ than those who received other vaccines before as an adult. The probability of COVID-19 vaccine uptake by those who had no history of contact with confirmed COVID-19 patients or clients was reduced by $58 \%$ [AOR $=0.42,95 \%$ CI: $0.20-0.86]$ compared to those who had direct contact with the confirmed COVID-19 patients or clients.

\section{Discussion}

This study assessed uptake of COVID-19 vaccine among health professionals and associated factors. The study found that $324(62.1 \%)$ of the health professionals were vaccinated for COVID-19 at least once. This finding was higher than the study finding from the Liverpool city council region which revealed the vaccine uptake of $51.4 \%{ }^{33}$ But, it was lower than the study findings in other developed countries such as New York City $(82 \%) .{ }^{34}$ The reason might be due to the increased morbidity and mortality rates in those countries, variation in the study periods and also due to the late arrival of the vaccine in Ethiopia. This study was also supported by a study conducted in low- and middle-income countries 
Table 2 Factors Associated with COVID-19 Vaccine Uptake Among Health Professionals in Ethiopia $(\mathrm{N}=522)$

\begin{tabular}{|c|c|c|c|c|c|}
\hline \multirow[t]{3}{*}{ Variables } & \multirow[t]{3}{*}{ Categories } & \multicolumn{2}{|c|}{ CoVID-19 Vaccine Uptake } & \multicolumn{2}{|c|}{ OR $[95 \% \mathrm{Cl}]$} \\
\hline & & Yes & No & COR & AOR \\
\hline & & $\mathrm{N}=324(62.1 \%)$ & $N=198(37.9 \%)$ & & \\
\hline \multirow[t]{4}{*}{ Age } & $18-29$ & $7(2.2 \%)$ & $13(6.6 \%)$ & 1 & \\
\hline & $30-39$ & $228(70.4 \%)$ & I 78 (89.9\%) & $2.38(0.93,6.08)$ & $0.92(0.22,3.78)$ \\
\hline & $40-49$ & $66(20.4 \%)$ & $5(2.5 \%)$ & $24.51(6.73,89.28)$ & $12.97(2.36,71.21)^{* *}$ \\
\hline & $50+$ & $23(7.1 \%)$ & $2(1.0 \%)$ & $21.36(3.85,48.35)$ & $18.95(2.04,36.29)^{*}$ \\
\hline \multirow[t]{2}{*}{ Sex } & Male & 305 (94.1\%) & $166(83.8 \%)$ & $3.09(1.70,5.63)$ & $2.91(1.05,8.09)^{*}$ \\
\hline & Female & $19(5.9 \%)$ & $32(16.2 \%)$ & 1 & \\
\hline \multirow[t]{2}{*}{ Resident } & Urban & $268(82.7 \%)$ & I 80 (90.9\%) & $0.48(0.27,0.84)$ & $0.78(0.32,1.89)$ \\
\hline & Rural & $56(17.3 \%)$ & $18(9.1 \%)$ & 1 & \\
\hline \multirow[t]{4}{*}{ Educational status } & College Diploma & $24(7.4 \%)$ & $4(2.0 \%)$ & 1 & \\
\hline & BA/BSc Degree & $142(43.8 \%)$ & $83(41.9 \%)$ & $0.28(0.09,0.85)$ & $0.27(0.05,1.34)$ \\
\hline & MA/MSc Degree & 101 (31.2\%) & $96(48.5 \%)$ & $0.17(0.05,0.52)$ & $0.48(0.08,2.59)$ \\
\hline & PHD and above & $57(17.6 \%)$ & $15(7.6 \%)$ & $0.63(0.19,2.10)$ & $1.26(0.20,7.76)$ \\
\hline \multirow[t]{5}{*}{ Monthly income } & $1651-3200$ & $6(1.9 \%)$ & 7 (3.5\%) & 1 & \\
\hline & $320 \mathrm{I}-5250$ & $35(10.8 \%)$ & $9(4.5 \%)$ & $4.54(1.22,16.87)$ & $4.64(0.47,45.57)$ \\
\hline & $5251-7800$ & $135(41.7 \%)$ & $37(18.7 \%)$ & $4.26(1.35,13.44)$ & $6.79(0.99,46.44)$ \\
\hline & $780 I-10,900$ & $88(27.2 \%)$ & $83(41.9 \%)$ & $1.24(0.39,3.83)$ & $2.54(0.36,17.68)$ \\
\hline & $>10,900$ & $60(18.5 \%)$ & $62(31.3 \%)$ & $1.13(0.36,3.55)$ & $1.01(0.14,7.48)$ \\
\hline \multirow[t]{6}{*}{ Place of work } & Hospital & $75(23.1 \%)$ & $39(19.7 \%)$ & $0.42(0.22,0.80)$ & $1.17(0.29,4.78)$ \\
\hline & Health Center & $90(27.8 \%)$ & $22(11.1 \%)$ & $0.89(0.45,1.79)$ & $1.76(0.43,7.26)$ \\
\hline & Academician & 59 (18.2\%) & $93(47.0 \%)$ & $0.14(0.07,0.25)$ & $1.55(0.29,8.11)$ \\
\hline & Private clinic & $6(1.9 \%)$ & $9(4.5 \%)$ & $0.15(0.05,0.46)$ & $0.23(0.04,1.40)$ \\
\hline & Others $^{a}$ & $12(3.7 \%)$ & 17 (8.6\%) & $0.16(0.06,0.38)$ & $0.31(0.07,1.39)$ \\
\hline & Health Offices & $82(25.3 \%)$ & $18(9.1 \%)$ & I & \\
\hline \multirow[t]{10}{*}{ Type of profession } & Nurse & $67(20.7 \%)$ & 57 (28.8\%) & $0.69(0.45,1.07)$ & $0.48(0.2 \mathrm{I}, \mathrm{I} .07)$ \\
\hline & Midwifery & $25(7.7 \%)$ & $15(7.6 \%)$ & $0.98(0.49,1.96)$ & $0.89(0.20,3.88)$ \\
\hline & Medical Doctor & 27 (8.3\%) & $3(1.5 \%)$ & $5.33(1.58,18.05)$ & $4.09(0.51,32.78)$ \\
\hline & Medical Laboratory & $6(1.9 \%)$ & $3(1.5 \%)$ & $1.19(0.29,4.85)$ & $0.43(0.02,12.40)$ \\
\hline & Pharmacy & $16(4.9 \%)$ & $6(3.0 \%)$ & $1.58(0.59,4.18)$ & $0.61(0.14,2.73)$ \\
\hline & Anesthesia & $3(0.9 \%)$ & $3(1.5 \%)$ & $0.59(0.117,2.99)$ & $3.63(0.17,76.35)$ \\
\hline & Psychiatry & $9(2.8 \%)$ & $9(4.5 \%)$ & $0.59(0.23,1.54)$ & $0.73(0.16,3.19)$ \\
\hline & Dentistry & $6(1.9 \%)$ & $3(1.5 \%)$ & $1.19(0.29,4.85)$ & $0.14(0.01,1.58)$ \\
\hline & Others ${ }^{b}$ & $3(0.9 \%)$ & $3(1.5 \%)$ & $0.59(0.12,2.99)$ & $1.37(0.14,13.49)$ \\
\hline & Public Health & $162(50.0 \%)$ & 96 (48.5\%) & I & \\
\hline
\end{tabular}


Table 2 (Continued).

\begin{tabular}{|c|c|c|c|c|c|}
\hline \multirow[t]{3}{*}{ Variables } & \multirow[t]{3}{*}{ Categories } & \multicolumn{2}{|c|}{ COVID-I9 Vaccine Uptake } & \multicolumn{2}{|c|}{ OR $[95 \% \mathrm{Cl}]$} \\
\hline & & Yes & No & COR & AOR \\
\hline & & $\mathrm{N}=324(62.1 \%)$ & $N=198(37.9 \%)$ & & \\
\hline \multirow[t]{5}{*}{ Staffing } & Academic staff & $67(20.7 \%)$ & $93(47.0 \%)$ & $0.19(0.12,0.33)$ & $0.23(0.10,0.49)^{* * *}$ \\
\hline & $\begin{array}{l}\text { Academic staffs working in } \\
\text { University Hospitals }\end{array}$ & II (3.4\%) & $12(6.1 \%)$ & $0.25(0.10,0.62)$ & $0.19(0.05,0.83)^{*}$ \\
\hline & Clinical staffs & $124(38.3 \%)$ & $59(29.8 \%)$ & $0.58(0.35,0.95)$ & $0.57(0.26,1.25)$ \\
\hline & Others $^{c}$ & $2(0.6 \%)$ & I $(0.5 \%)$ & $0.55(0.05,6.26)$ & $0.06(0.002,1.77)$ \\
\hline & Health Offices staffs & $120(37.0 \%)$ & $33(16.7 \%)$ & 1 & \\
\hline \multirow[t]{2}{*}{ Perceived own health status } & Not healthy & $53(16.4 \%)$ & 44 (22.2\%) & 1 & \\
\hline & Healthy & $27 \mid(83.6 \%)$ & $154(77.8 \%)$ & $1.46(0.94,2.28)$ & $0.73(0.25,2.12)$ \\
\hline \multirow[t]{2}{*}{ Perceived family health status } & Not-Healthy & 69 (21.3\%) & 70 (35.4\%) & I & \\
\hline & Healthy & 255 (78.7\%) & $128(64.6 \%)$ & $2.02(1.36,2.99)$ & $4.4(2.21,8.75)^{* * *}$ \\
\hline \multirow[t]{2}{*}{ Ever been tested } & Yes & 155 (47.8\%) & 54 (27.3\%) & I & \\
\hline & No & $169(52.2 \%)$ & 144 (72.7\%) & $2.45(1.67,3.56)$ & $1.50(0.79,2.83)$ \\
\hline \multirow[t]{3}{*}{ Chronic disease for respondent } & Yes & $35(10.8 \%)$ & $13(6.6 \%)$ & 1 & \\
\hline & No & $270(83.3 \%)$ & I 73 (87.4\%) & $0.58(0.29,1.13)$ & $0.87(0.26,2.83)$ \\
\hline & Not sure & $19(5.9 \%)$ & $12(6.1 \%)$ & $0.59(0.224,1.54)$ & $0.57(0.08,3.87)$ \\
\hline \multirow{2}{*}{$\begin{array}{l}\text { History of receiving other } \\
\text { vaccine before as an adult }\end{array}$} & Yes & $270(83.3 \%)$ & 137 (69.2\%) & 1 & \\
\hline & No & $54(16.7 \%)$ & $6 \mathrm{I}(30.8 \%)$ & $2.23(1.46,3.39)$ & $4.07(2.07,8.01)^{* * * *}$ \\
\hline \multirow{2}{*}{$\begin{array}{l}\text { Ever involved in isolation center } \\
\text { or care }\end{array}$} & Yes & $72(22.2 \%)$ & $27(13.6 \%)$ & $1.81(1.12,2.93)$ & $0.55(0.17,1.67)$ \\
\hline & No & $252(77.8 \%)$ & |7| (86.4\%) & 1 & \\
\hline \multirow{3}{*}{$\begin{array}{l}\text { History of contact with } \\
\text { confirmed patients or clients }\end{array}$} & Yes, I have direct contact & 81 (25.0\%) & 44 (22.2\%) & I & \\
\hline & Yes, but no direct contact & 89 (27.5\%) & $34(17.2 \%)$ & $1.42(0.83,2.44)$ & $0.89(0.37,2.15)$ \\
\hline & No contact & 154 (47.5\%) & $120(60.6 \%)$ & $0.69(0.45,1.08)$ & $0.42(0.20,0.86)^{*}$ \\
\hline
\end{tabular}

Notes: *P-value $<0.05$, **P-value $<0.0 \mathrm{I}$, ***P-value $<0.00 \mathrm{I}$ and I=reference, ${ }^{\mathrm{a} N o n-G o v e r n m e n t a l ~ O r g a n i z a t i o n, ~ B l o o d ~ b a n k, ~}{ }^{\mathrm{b}}$ Integrated Emergency surgery and Obstetrics, Health Education, Environmental health professional's 'Non-Governmental Organization staffs.

Abbreviations: AOR, Adjusted odds ratio; COR, Crude Odds Ratio; $\mathrm{Cl}$, Confidence Interval.

(LMIC) which indicated that COVID-19 vaccine acceptance across LMIC ranged from $66.5 \%$ to $96.6 \%$ with an average of $80.3 \%$. Of which, health-care workers in Sierra Leone have shared greater acceptance. ${ }^{35}$

Age, sex, profession, perceived family health status, previous experiences of receiving other vaccines and history of contact with a COVID-19 client were found to be predictors of COVID-19 vaccine uptake in this study. In this regard, male health professionals were 2.9 times $(\mathrm{AOR}=2.91$; CI: $1.05,8.09, \mathrm{p}=0.041)$ more likely to uptake COVID-19 vaccine than female health professionals. This finding was in line with the studies conducted in the Democratic Republic of Congo, ${ }^{36}$ Ghana, ${ }^{37}$ United States of America $^{38}$, Kingdom of Saudi Arabia and Uganda. ${ }^{17,39,40}$ The variation might arise from the fear of a vaccine shot by female health professionals. The difference might also be attributed to the large proportion of males than females proportion in this study.

The study also found that COVID-19 vaccine uptake was increased among older ages (40-49 and 50+) compared to those 18-29 years. This finding was similar with the study done in United States of America, Bangladesh and Kingdom of Saudi Arabia. ${ }^{17,38,41,42}$ This might be due to the fact that there is an increment in understanding of 
COVID-19 risk among elders. But, the study contradicts with the finding of the study conducted in Bangladesh where young people took the vaccine more than old people. $^{43}$ The dissimilarity might be attributed to the difference in the level of awareness and variation in the study population. But, in some of the studies, age had not been associated with the COVID-19 vaccine uptake. ${ }^{36,37,40}$

In addition, the study found that respondents who were academic staff working in the teaching institutions were less likely to uptake COVID-19 vaccine than those who worked in the clinical area. There was no study that assessed the association of this factor with the COVID19 vaccine uptake before. However, the reason might be due to the fact that clinical staffs are more at risk for COVID-19 than academic staffs.

The study also showed that perceived family health status was found to be the significantly associated factor for COVID-19 Vaccine uptake. Health workers who perceived their families as healthy were 4.4 times more likely to uptake COVID-19 vaccine than those who perceived their families as not healthy. But, this finding contradicts with the study conducted in Bangladesh. ${ }^{43}$ The reason might be due to fear of vaccine side effects on unhealthy people, to protect healthy people from diseases, and participants' low confidence in the health-care system in developing countries including Ethiopia. Also, it was supported by a study conducted in Pakistan. ${ }^{44}$

The study also revealed that experience of ever receiving other vaccines was found to be one of the factors significantly associated with COVID-19 vaccine uptake. It indicated that those health professionals who have no history of receiving other vaccines as an adult were almost 4 times more likely to uptake COVID-19 vaccine than their counterparts. This contradicts the finding from prior systematic review in which previous history of flu vaccination was associated with COVID-19 vaccination acceptance. ${ }^{15}$ However, it was comparable with the study conducted in Bangladesh in which previous history of vaccination was negatively associated with COVID-19 vaccine uptake. ${ }^{42}$ The possible reason might be because of fear of side effects among those who have a history of receiving other vaccines as an adult.

In this study, history of contact with clients or patients of COVID-19 case was found to be one of the factors significantly associated with COVID-19 vaccine uptake which was similar with the finding of the study done in Ghana. ${ }^{37}$ The possible reason might be due to more risk perception among health professionals having history of contact with COVID-19 case than their counterparts.

This study has its own limitations. First, the cross-sectional nature of study design, which prevents identification of causal factors. Second, this study used non-probability sampling techniques, snowball, which limits the extent to which the results could be generalized to the population of health professionals in the study area. Third, health professionals who had no access to the internet services could not participate in this study. Fourth, an online survey could lead to a low response rate. Regardless of these limitations, this study highlights COVID-19 vaccine uptake and associated factors, which will be an input for decision makers, policy designers, implementers, and managers of health service organizations from bottom to top level to increase the uptake of the vaccine.

\section{Conclusion}

The study found that COVID-19 vaccine uptake among health professionals was low. This was not sufficient to achieve herd immunity as at least nine out of ten health professionals are required for herd immunity ${ }^{45}$ in order to reduce the transmission of the disease pandemic.

Health workforces are one of the critical components of health systems building block in Ethiopian health sector reform and are a reliable source of health information and their uptake of COVID-19 vaccines can influence the general population. As COVID-19 pandemic is currently a major threat to public health and has had a significant impact on the overall general public, high vaccine uptake has its own clinical and public health implication. Age, sex, place of work, perceived family health status, previous experience of receiving a vaccine as an adult and contact history with COVID19 clients or patients were the factors that influence the COVID-19 vaccine uptake among health professionals in Ethiopia. Hence, the decision makers and health managers in collaboration with different institutions and stakeholders should consider instituting mandatory vaccination for health professionals and design strategies for the provision of the vaccine. Besides this, further studies need to be conducted to determine the economic cost of COVID-19 vaccine from the provider's perspective.

\section{Acknowledgments}

Researchers would like to acknowledge all participants of the study and Wollega University for their due cooperation and involvement. 


\section{Author Contributions}

All authors made substantial contributions to conception and design, acquisition of data, or analysis and interpretation of data; took part in drafting the article or revising it critically for important intellectual content; gave final approval of the version to be published; and agree to be accountable for all aspects of the work.

\section{Disclosure}

All authors declared that they have no conflicts of interest related to this work.

\section{References}

1. Galveston BS. Structure and classification of viruses. In: Medical Microbiology. 4th ed. University of Texas Medical Branch at Galveston; 1996.

2. Sim M. The COVID-19 pandemic: major risks to healthcare and other workers on the front line. Occup Environ Med. 2020;77:281-282. doi:10.1136/oemed-2020-106567

3. Euro Surveillance Editorial Team. Note from the editors: World Health Organization declares novel coronavirus (2019 nCoV) sixth public health emergency of international concern. Euro Surveill. 2020;25(5):200131e.

4. Johns Hopkins Uneversity. COVID-19 Data in Motion: Available from: https://coronavirusjhuedu/. Accessed October 5, 2021.

5. Word Health Organization. Africa/first case of COVID-19 confirmed in Ethiopia. Available from: https://www.afro.who.int/news/first-case -covid-19-confirmed-ethiopia. Accessed June 28, 2021.

6. Ethiopian Public Health Institute Homepage. Quick updates on COVID-19. Available from: https://ephi.gov.et/. Accessed June 28, 2021.

7. Adaptive phase IB-II randomized clinical trial of preventive vaccine consisting of autologous dendritic cells loaded with antigens from severe acute respiratory syndrome Coronavirus-2 (SARS-CoV-2), with or without GM-CSF, in subjects negative for COVID-19 infection and anti-SARS-CoV-2 antibodies. ClinicalTrials.gov identifier (NCT number): NCT04386252. Available from: https://clinicaltrials. gov/ct2/show/NCT04386252. Accessed June 2, 2021.

8. A randomized, double-blind, placebo-controlled phase 3 study to assess the efficacy and safety of Ad26.COV2.S for the prevention of SARS-CoV-2-mediated COVID-19 in adults aged 18 years and older. ClinicalTrials.gov Identifier: NCT04505722. Available from: https:// clinicaltrials.gov/ct2/show/NCT04505722. Accessed June 28, 2021.

9. Tracking Coronavirus vaccinations around the world by Josh Holder. Available from: https://wwwnytimescom/interactive/2021/world/ covid-vaccinations-trackerhtml. Accessed October 5, 2021.

10. WHO. Ethiopia Introduces COVID-19 Vaccine in a National Launching Ceremony. WHO; 2021.

11. WHO. Monitoring COVID-19 vaccination: considerations for the collection and use of vaccination data, interim guidance. 3 March 2021. Available from https://www.who.int/publications $/ \mathrm{i} / \mathrm{item} / \mathrm{moni}$ toring-covid-19-vaccination-interim-guidance?_cf_chl_managed_ tk_=EodgaZSVnUfrBh6HVwCuHngo57MAqcZ_aMDyQ333dm41639551661-0-gaNycGzNCT0

12. National Academies of Sciences, Engineering, and Medicine. Framework for equitable allocation of COVID-19 vaccine. 2020.

13. Centers for Disease Control and Prevention. How CDC is making COVID-19 vaccine recommendations. 2020.

14. World Health Organization. WHO SAGE Roadmap for Prioritizing Uses of COVID-19 Vaccines in the Context of Limited Supply. World Health Organization; 2020.
15. Galanis PA, Vraka I, Fragkou D, Bilali A, Kaitelidou D. Intention of health care workers to accept COVID-19 vaccination and related factors: a systematic review and meta-analysis. medRxiv Preprint. 2020. doi: $10.1101 / 2020.12 .08 .20246041$

16. Chor JSY, Ngai KLK, Goggins WB, et al. Willingness of Hong Kong healthcare workers to accept pre-pandemic influenza vaccination at different WHO alert levels: two questionnaire surveys. BMJ. 2009;339:b3391. doi:10.1136/bmj.b3391

17. Biswas N, Mustapha T, Khubchandani J, et al. The nature and extent of COVID-19 vaccination hesitancy in healthcare workers. $J$ Commun Health. 2021;46:1244-1251. doi:10.1007/s10900-021-00984-3

18. Nzaji MK, Ngombe LK, Mwamba GN, et al. Acceptability of vaccination against COVID-19 among healthcare workers in the Democratic Republic of the Congo pragmatic and observation research. Lancet Glob Health. 2020;8:e1455-e1456. doi:10.1016/ S2214-109X(20)30454-X

19. Belsti Y, Gela YY, Akalu Y, et al. Willingness of Ethiopian population to receive COVID-19 vaccine. $J$ Multidiscip Healthc. 2021;14:1233-1243. doi:10.2147/JMDH.S312637

20. Hamel L, Kirzinger A, Muñana C, Brodie M. KFF COVID-19 vaccine monitor; 2020. Available from: https://www.kff.org/coronavir uscovid19/report/kff-covid-19-vaccine-monitor-december-2020/. Accessed December 9, 2021.

21. Gharpure R, Guo A, Bishnoi CK, et al. Early COVID 19 first-dose vaccination coverage among residents and staff members of skilled nursing facilities participating in the pharmacy partnership for long-term care program- United States, December 2020-January 2021. Morbid Mortal Wkly Rep. 2021;70(5):178. doi:10.15585/mmwr.mm7005e2

22. World Health Organization. WHO SAGE Values Framework for the Allocation and Prioritization of COVID-19 Vaccination. World Health Organization; 2020.

23. Aljazeera News/Corona virus Pandemic. At least 17,000 health-workers have died from COVID: Amnesty, March 5,2021. Available from: https://www.aljazeera.com/news/2021/3/5/at-least-17000-healthworkers-have-died-from-covid-amnesty. Accessed December 9, 2021.

24. Piccirillo B, Gaeta T. Survey on use of and attitudes toward influenza vaccination among emergency department staff in a New York metropolitan hospital. Infect Control Hosp Epidemiol. 2006;27(6):618-622. doi: $10.1086 / 504448$

25. Vasilevska M, Ku J, Fisman D. Factors associated with healthcare worker acceptance of vaccination: a systematic review and meta-analysis. Infect Control Hosp Epidemiol. 2014;35(6):699-708. doi:10.1086/676427

26. Heininger U, Bächler M, Schaad U. Attitudes of pediatricians regarding influenza selfimmunization: a survey in a Swiss university children's hospital. Pediatr Infect Dis J. 2003;22(5):391-394. doi:10.1097/01.inf.0000066901.59298.a8

27. Aguilar-Díaz FDC, Jiménez-Corona ME, Ponce-de-león-rosales S. Influenza vaccine and health care workers. Arch Med Res. 2011;42 (8):652-657. doi:10.1016/j.arcmed.2011.12.006

28. Takahashi O, Noguchi Y, Rahman M, et al. Influence of family on acceptance of influenza vaccination among Japanese patients. Fam Pract. 2003;20(2):162-166. doi:10.1093/fampra/20.2.162

29. Fiebach N, Viscoli C. Patient acceptance of influenza vaccination.. Am J Med. 1991;91(4):393-400. doi:10.1016/00029343(91)90157-S

30. World Health Organization. Behavioural considerations for acceptance and uptake of COVID-19 vaccines: WHO technical advisory group on behavioural insights and sciences for health, meeting report; 2020. World Health Organization. Available from: https://apps.who. int/iris/handle/10665/337335. Accessed December 9, 2021.

31. Ethiopia Federal Ministry of Health (MOH). HSTP health sector transformation plan 2015/16-2019/20 (2008-2012 EFY); 2015. Available from: https://wwwglobalfinancingfacilityorg/ ethiopia-health-sector-transformation-plan-201516-201920. Accessed December 1, 2021. 
32. COVID-19 vaccine uptake among health care workers. Evidence synthesis briefing note. Available from: https://esnetworkca/brief ings/covid-19-vaccine-uptake-among-health-care-workers/. Accessed December 1, 2021.

33. Tulloch JSP, Lawrenson K, Gordon AL, et al. COVID-19 vaccine hesitancy in care home staff: a survey of Liverpool care homes. medRxiv. 2021. doi:10.1101/2021030721252972

34. Baniak LM, Luyster FS, Raible CA, et al. COVID-19 vaccine hesitancy and uptake among nursing staff during an active vaccine rollout. Vaccines. 2021;9:858. doi:10.3390/vaccines9080858

35. Arce JSS, Warren SS, Meriggi NF, et al. COVID-19 vaccine acceptance and hesitancy in low- and middle-income countries. Nat Med. 2021;27:1385-1394. doi:10.1038/s41591-021-01454-y

36. Nzaji MK, Ngombe LK, Mwamba GN, et al. Acceptability of vaccination against COVID-19 among healthcare workers in the Democratic Republic of the Congo. Pragmat Observ Res. 2020;11:103-109. doi:10.2147/POR.S271096

37. Agyekum MW, Afrifa-Anane GF, Kyei-Arthur F, et al. Acceptability of COVID-19 vaccination among health care workers in Ghana. Hindawi. 2021;2021:8. doi:10.1155/2021/9998176

38. Shekhar R, Sheikh AB, Upadhyay S, et al. COVID-19 vaccine acceptance among health care workers in the United States. Vaccines. 2021;9:119. doi:10.3390/vaccines9020119

39. Kanyike AM, Olum R, Kajjimu J, et al. Acceptance of the coronavirus disease-2019 vaccine among medical students in Uganda. Trop Med Health. 2021;49:37. doi:10.1186/s41182-021-00331-1
40. Qattan AMN, Alshareef N, Alsharqi O, et al. Acceptability of a COVID-19 vaccine among healthcare workers in the Kingdom of Saudi Arabia. Front Med. 2021;8. doi:10.3389/fmed.2021.644300

41. Gerussi V, Peghin M, Palese A, et al. Vaccine hesitancy among Italian patients recovered from COVID-19 infection towards influenza and Sars-Cov-2 vaccination. Vaccines. 2021;9:172. doi:10.3390/ vaccines 9020172

42. Mahmud S, Mohsin M, Khan IA, et al. Acceptance of COVID-19 vaccine and its determinants in Bangladesh. arXiv. 2021;7:e07376.

43. Abedin M, Islam MA, Rahman FN, et al. Willingness to vaccinate against COVID-19 among Bangladeshi adults: understanding the strategies to optimize vaccination coverage. PLoS One. 2021;16(4): e0250495. doi:10.1371/journal.pone.0250495

44. Cascinia F, Pantovicb A, Al-Ajlounic Y, et al. Attitudes, acceptance and hesitancy among the general population worldwide to receive the COVID-19 vaccines and their contributing factors: a systematic review. EClinicalMedicine. 2021;40:101113. doi:10.1016/j. eclinm.2021.101113

45. Anderson MD, Merkin SS, Everson-Rose SA, et al. Health literacy within a diverse community-based cohort: the multi-ethnic study of atherosclerosis. J Immigr Minor Health. 2020;23:659-667. doi:10.1007/s10903-020-01123-1
Infection and Drug Resistance

\section{Publish your work in this journal}

Infection and Drug Resistance is an international, peer-reviewed openaccess journal that focuses on the optimal treatment of infection (bacterial, fungal and viral) and the development and institution of preventive strategies to minimize the development and spread of resistance. The journal is specifically concerned with the epidemiology of
Dovepress

antibiotic resistance and the mechanisms of resistance development and diffusion in both hospitals and the community. The manuscript management system is completely online and includes a very quick and fair peerreview system, which is all easy to use. Visit http://www.dovepress.com/ testimonials.php to read real quotes from published authors. 